\title{
The Flow Simulation in the Fluidic Amplifier
}

\author{
Yong $\mathrm{Xu}^{1}{ }^{1}$, Guoqing $\mathrm{Zhang}^{1}$ and Fei Wang ${ }^{2}$ \\ ${ }^{1}$ Key Laboratory of Dynamics and Control of Flight Vehicle, Ministry of Education, School of Aerospace Engineering, \\ Beijing Institute of Technology, Beijing, 100081, China \\ ${ }^{2}$ School of Mechanical \& Electronic Engineering, Beijing Institute of Technology, Beijing, 100081, China
}

\begin{abstract}
The viscous flow in the fluidic amplifier is simulated numerically based on CFD technology, and many tests have been done to verify the numerical precision. The results show that the calculated data are good agreement with the experimental data. So the numerical simulation of the viscous flow in the fluidic amplifier is accurate and reliable, and it can be applied to investigate the steady flow and unsteady flow in the fluidic amplifier.
\end{abstract}

Keywords: Fluidic amplifier, Flow simulation, Size function, Switching time.

\section{INTRODUCTION}

On one hand, the fluidic amplifier can be used as the logic control system components to achieve a variety of logic functions, on the other hand, through a small energy control signals, it also could be served as an implementation part of the control system and offer much large energy or torque directly [1]. Nowadays the fluidic amplifier is applied to the Multiple Launch Rocket System project as the implemental components of the control system [2], such as the "tornado" weapon system of Russia. At the beginning of this subject, based on the model development and test, we have done many tests, including a variety of geometric shapes, main gas supply pressures, as well as a variety of control flow flux, which measure the outputting thrust and switching time.

\section{EXPERIMENTAL PROGRAM DESIGN}

Both of the validation of the thrust in the attached wall condition and the verification of the switching time in switching process are completed by the single-channel air test system [3]. This test system consists of high pressure and large flow of the pressure regulator system, data acquisition and processing system, moving frame of the jet components as well as the control components. The Fig. (1) has shown the schematic diagram of the fluidic amplifier test system. The high pressure and large flow of the pressure regulator system includes a high-pressure compressor (WZ2.3/450) and a high pressure cylinder group (TGP$50 \mathrm{~L} / 35 \mathrm{Mpa}$ ), which mainly provide the high pressure gas source and control flow for the fluidic amplifier. The moving frame is used to fix the fluidic amplifier, on which we would install two high frequency high-precision force sensors to detect the size of a combined thrust and thrust switch changes in the course of joint of the mobile frame. The data

*Address correspondence to this author at the School of Aerospace Engineering, Beijing Institute of Technology, Beijing, 100081, China;

Tel: +86-010-68918171; Fax: +86-010-68911040;

E-mail: xyxuyong@bit.edu.cn

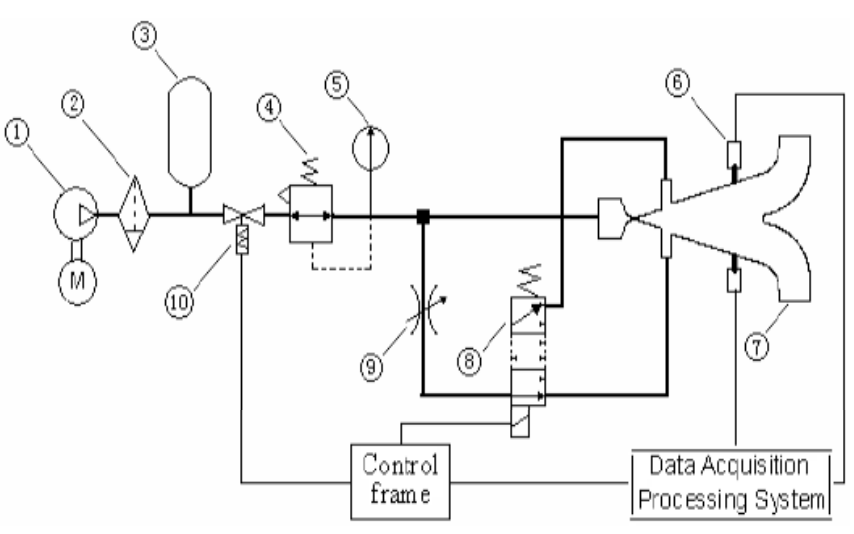

Fig. (1). The schematic diagram of the fluidic amplifier test system.

(1)-Air Compressors, (2)-Filter, (3)-Air Cylinder, (4)-Decompression Pressure Valve, (5)-Pressure Table, (6-Force Sensor, (7)-Fluidic Amplifier, 8- Three Links Directional Electromagnetic Control Valve, (9)-Adjustable Throttle, (10-Cutoff Value.

acquisition and processing system are used to collect the voltage signal coming from the collection force sensor [4].

\section{Numerical Simulation Method}

Fig. (2) has shown the solid scheme of the fluidic amplifier, and Fig. (3) has shown the surface girds of the fluidic amplifier. In modeling the whole fluidic amplifier, the quality and size of the mesh generation would often directly affect the accuracy of the experimental results. But the key technology which affected the quality and quantity of the grids generation is to control and grasp size of the grid. Based on this, during meshing the structural grids of the fluidic amplifier, the experiment took the method of size function control grid size (It doesn't change the entire structure of the network topology) [5]. And the parts of structural grid were refined for the whole device, and the structural grid had been smoothed and swapped, enhancing the accuracy of the experiment. The Gauss-Seidel method [6] is used to calculate the inner fluid field of the fluidic amplifier. The govern- 


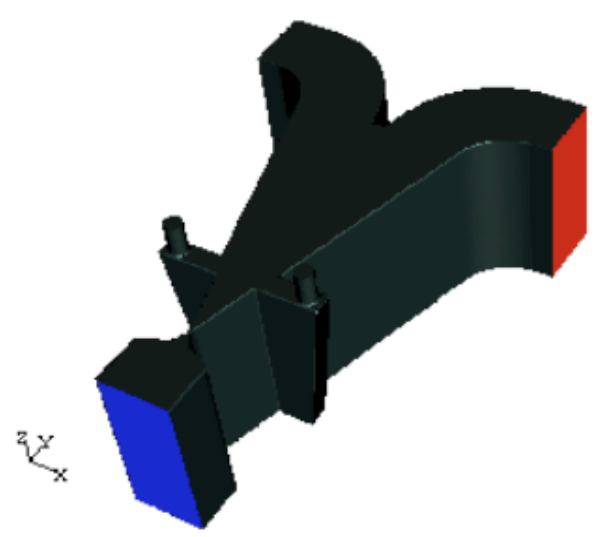

Fig. (2). The solid scheme of the fluidic amplifier

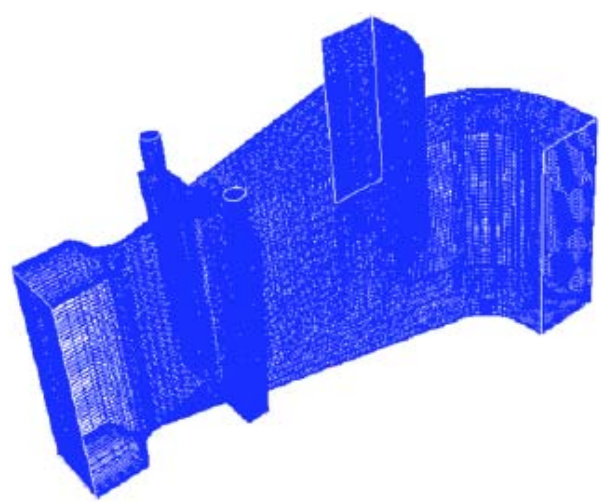

Fig. (3). The surface grids of the fluidic amplifier.

ing equations of flow are Reynolds Averaging Navier-Stokes equation [7]. The model of turbulence is Realizable kepsilon model $[8,9]$, and Two-layer zonal model is used near the wall [10-12].

\section{Size Function}

As shown in Fig. (4), in the field of unstructured grid, to control and master the grid size is the key technology for the mesh quality. Usually there are three ways to control the mesh size. The first is distributing notes on the border to control the mesh size, for complex surfaces, this method is only used to increase the number of the grid to ensure the authenticity of the grid and improve the mesh quality. The second is through micro modifying the local grid, however, due to the limits of part of girds, improving the quality of the grid is not applicable in all cases. The third way is to use the size function, which can be easily determined the distribution of the grid based on geometric and physical properties. The most common method is through the grid background to define the function of the grid cell size (The issue raised by S. Pirzadeh).

The unstructured gird of the Fluidic amplifier is used the custom size and curvature size function. Custom Size function is a constant user-defined grid size, and curvature size function definition is used to obtain the maximum angle of the two adjacent grid surfaces, which can accurately use the grid to construct the surface. The size of grid is defined as follows:

$S_{n}=2 * \sin \left(\theta_{\max } / 2\right) / \rho_{\max }$

Where $\rho_{\max }$ is the maximum curvature along two orthogonal axes, which uses the size function to provide information for the background of grid.

In the process of unstructured grid generation, there will be a lot of unpredictability and opacity, therefore, the quality of the grid must be detected. The most popular detection methods are isometric folding rate law and two sides folding rate method.

$Q_{E A S}$ is the folding rate of isometric, $\theta_{\max }$ and $\theta_{\min }$ is respectively the minimum and maximum angle between the grid cells.

$Q_{E A S}=\max \left\{\frac{\theta_{\max }-\theta_{e q}}{180-\theta_{e q}}, \frac{\theta_{e q}-\theta_{\min }}{\theta_{e q}}\right\}$

Folding rate of grid size $Q_{E A S}$

$Q_{E A S}=\frac{S_{e q}-S}{S_{e q}}$

Where $S$ is the grid cell area or volume, $S_{e q}$ is the area or volume composed by the longest side of the grid cells.

\section{The Validation of the Thrust in the Attached Wall Condition}

The thrust in the attached wall condition is one important measurement to indicate the performance of supersonic jet components good or bad. Therefore, we would first calculate the pressure thrust created by a variety of primary gas source pressure, and then under the same conditions, we would obtain the thrust value corresponding to the main gas source
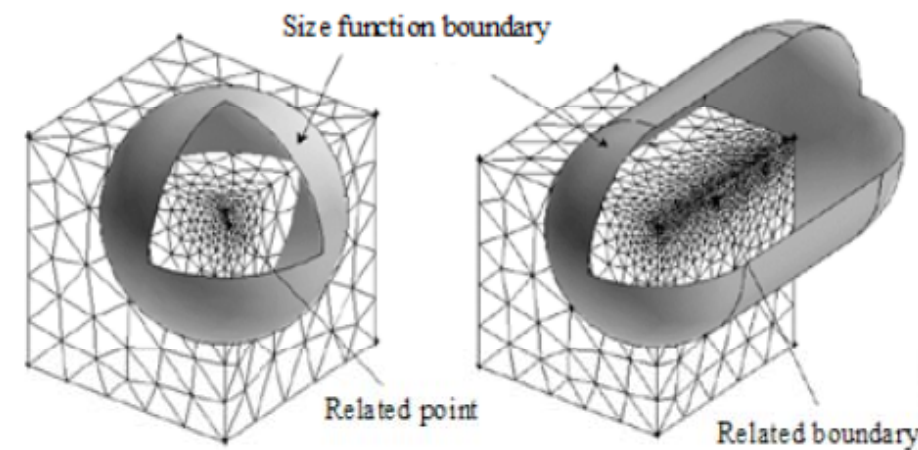

$\theta_{\max }$

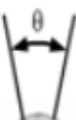


Table 1. The Calculated and the Experimental Values of Thrusts

\begin{tabular}{|c|c|c|c|c|c|}
\hline Ps (bar) & Test (N) & $\mathbf{f}_{\mathbf{r}}(\mathbf{N})$ & $\mathbf{f}_{\mathbf{l}}(\mathbf{N})$ & $\mathbf{F}(\mathbf{N})$ & Error $(\%)$ \\
\hline 30 & 79.9 & 84.34 & 1.95 & 82.40 & 3.13 \\
\hline 40 & 106.0 & 108.91 & 4.43 & 104.48 & -1.43 \\
\hline 45 & 126.3 & 142.09 & 4.58 & 137.51 & 8.87 \\
\hline 50 & 142.6 & 164.59 & 5.66 & 158.93 & 11.45 \\
\hline 60 & 179.3 & 186.87 & 6.43 & 180.44 & 0.64 \\
\hline 70 & 202.8 & 220.68 & 8.55 & 212.12 & 4.6 \\
\hline 80 & 239.9 & 263.05 & 18.04 & 245.01 & 2.1 \\
\hline 90 & 275.4 & 296.36 & 21.78 & 274.58 & -0.3 \\
\hline
\end{tabular}

pressure. Table 1 has showed the thrust corresponding to the main gas source pressure (Ps) and test stands for the total thrust in the $\mathrm{X}$ direction of the jet components. $\mathrm{f}_{\mathrm{l}}$ and $\mathrm{f}_{\mathrm{r}}$ stand for their own thrusts of two outlets, $\vec{F}=\vec{f}_{l}+\vec{f}_{r}$, Error stands for the relative error between the calculation ( $F$ ) and test.

\section{The Verification of the Switching Time in Switching Process}

The switching time is another important measurement to indicate the performance of supersonic jet components good or bad. We defined the switching time as from the beginning of the control flow changes to the reverse thrust on output became to be stable. In the experiment, the control signal is changed by changing the role of the control signals on the implementation of the electromagnet. Since the change from the control solenoid to control the flow signal changes in response between the solenoid armatures, pneumatic amplification mechanism, air flow through the control of channel and other areas of the delay, which is difficult to determine the starting of the switching time. Therefore, this research would be carried out by comparing the thrust rise time and thrust falling time obtained by calculating and experiment, which could verify the correctness of the unsteady calculation.

\section{RESULT}

\section{Calculation Result}

Shown in Fig. (5), Ps=100bar, $\mathrm{m}_{\mathrm{l}}=0.1 \mathrm{~kg} / \mathrm{s}, \mathrm{m}_{\mathrm{r}}=0.003 \mathrm{~kg} / \mathrm{s}$, and the thrust rise time is $1.11 \mathrm{~ms}$, the thrust falling time is $1.23 \mathrm{~ms}$.

\section{Experiment Result}

Shown in Fig. (6), it is the thrust change during switching waveforms in experimental value. Among them, the top of the square wave stand for the input control signal, the below purple curve is the combined thrust on output measured by two force sensors, the green curve is to eliminate noise and smooth thrust after the output [4]. The thrust rise time is $1.18 \mathrm{~ms}$, and the thrust falling time is $0.85 \mathrm{~ms}$.
The Parameters Changes of the Right and Left Output

The Static Pressure of Right Output, Density, X Direction Velocity and Static Temperature Changes

As shown in Fig. (7), when $\mathrm{t}=3.56 \mathrm{~ms}$, although export static pressure, density and temperature significantly higher than other times of the static pressure, density and temperature, But the X direction velocity is clearly slower than other moments. For the thrust is proportional to the square of $\mathrm{X}$ direction velocity, therefore, the thrust generated by the right output has reached to the minimum at this time. When $\mathrm{t}=4.08 \mathrm{~ms}$, the $\mathrm{X}$ direction velocity of right output has increased to the peak, so the thrust has reach to maximum. Compared with the flow parameters $(\mathrm{t}=3.84 \mathrm{~ms}$ VS $\mathrm{t}=4.08 \mathrm{~m})$, whose $\mathrm{X}$ direction velocity is quite identical. However, the density and static pressure of the former are relatively smaller, so the thrust $(\mathrm{t}=3.84 \mathrm{~ms})$ is smaller than the thrust $(\mathrm{t}=4.08 \mathrm{~ms})$.

\section{The Static Pressure of Left Output, Density, X Direction Velocity and Static Temperature Changes}

From Fig. (8) we can see, when $\mathrm{t}=3.56 \mathrm{~ms}, \mathrm{t}=3.68 \mathrm{~ms}$, $\mathrm{t}=3.84 \mathrm{~ms}, \mathrm{t}=4.08 \mathrm{~ms}$, the left output has create the back eddy. Compared with the Fig. (7) and Fig. (8), the parameters change of the right output shows relatively routine, and the left output shows chaotic. It is mainly due to that the left output flow is not only disturbed by the main vortex, but also disturbed by the back eddy as well as two unsteady vortexes on the concave left wall.

\section{CONCLUSIONS}

From Table 1 we can see that the calculated thrusts showed the agreement with the experimental test, especially when the Ps> 50bar, the relative error is below 5\%. Comparing Fig. (5) with Fig. (6), we can see that the thrust rise time and the rising time in calculation result is similar to the thrust rise time and the rising time in experiment result. So the numerical simulation of the viscous flow for the fluidic amplifier is accurate and reliable, and it can be applied to investigate the steady flow and unsteady flow in fluidic amplifier. 


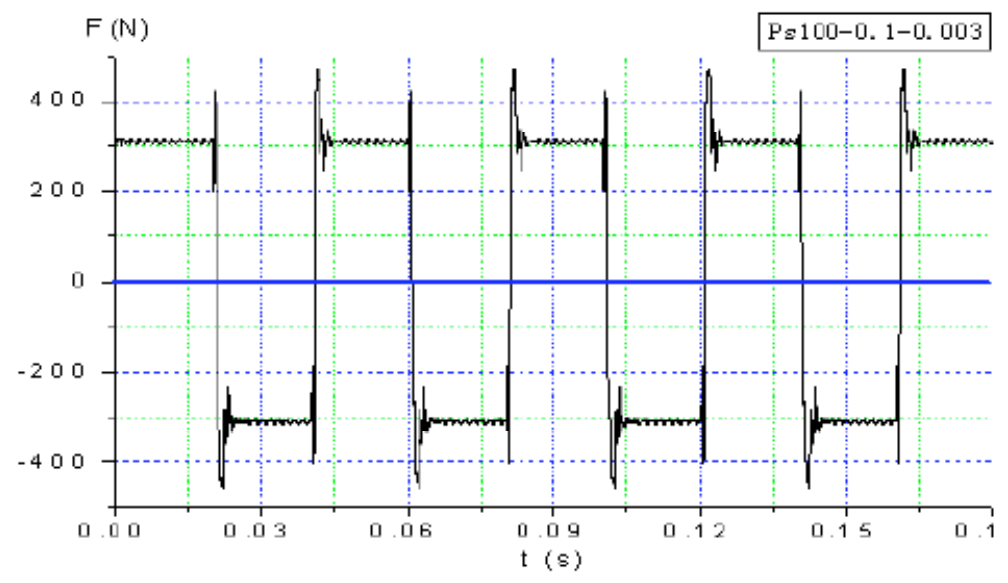

Fig. (5). Thrust change during switching waveforms (calculated value).

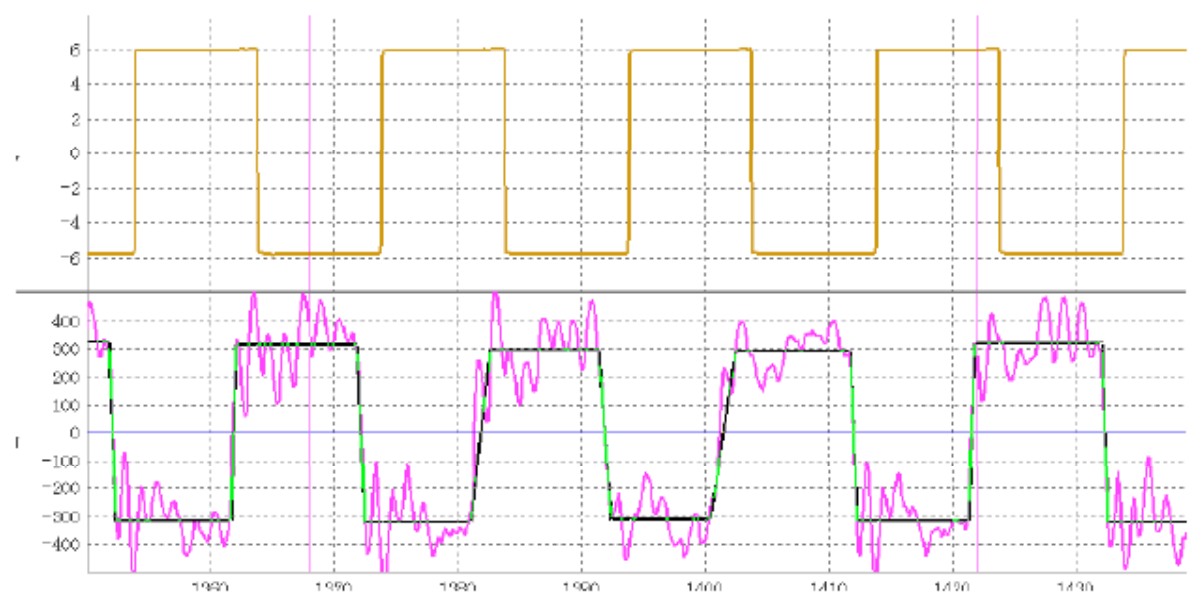

Fig. (6). Thrust change during switching waveforms (experimental value).

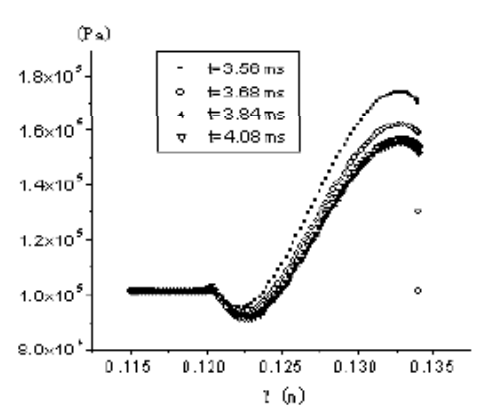

(a) The static pressure of right output

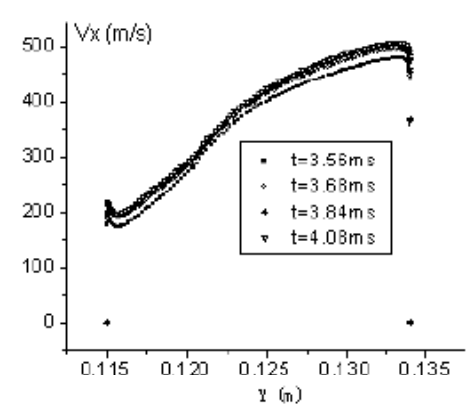

(c) $\mathrm{X}$ direction velocity

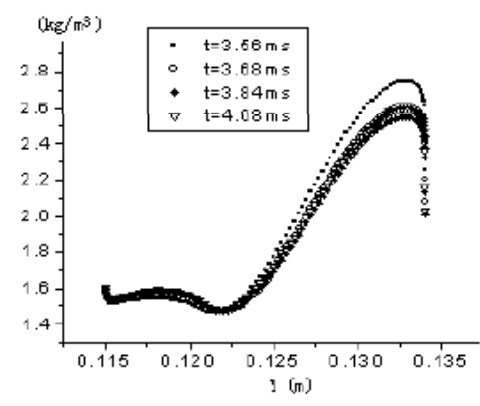

(b) density

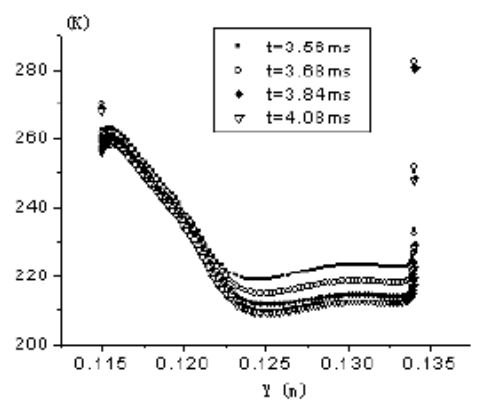

(d) static temperature changes

Fig. (7). The parameters distribution changes of the right output. 


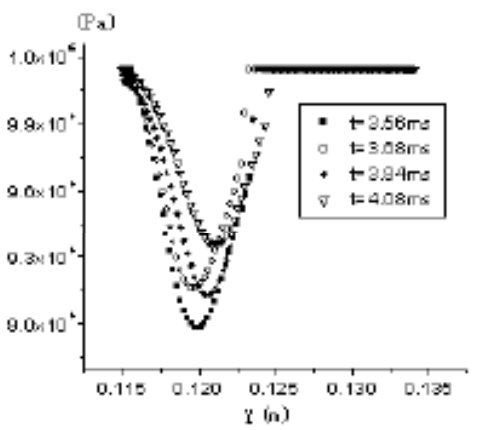

(a) The static pressure of left output

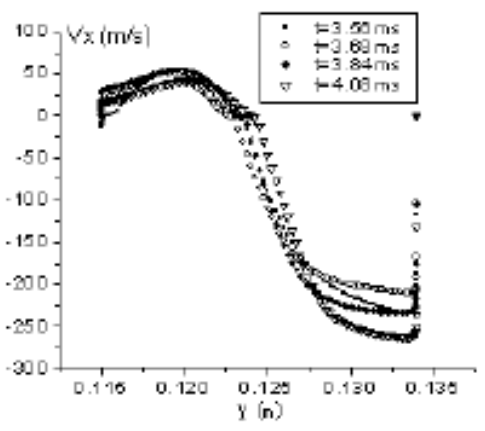

(c) $\mathrm{X}$ direction velocity

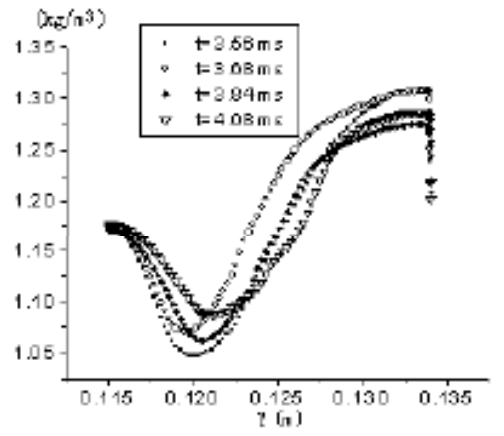

(b) density

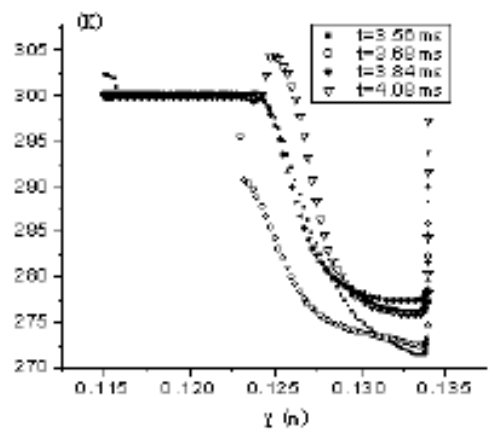

(d) static temperature changes

Fig. (8). The parameters distribution changes of the left output.

\section{ACKNOWLEDGMENTS}

This research was supported by the Natural Science Foundation of China (10972034).

\section{CONFLICT OF INTEREST}

None declared.

\section{REFERENCES}

[1] Y. Xu, S.X. Yang, Z.G. Zhou, In: Proceedings of the Fifth International Conference on Fluid Power Transmission and Control (2001).

[2] F. Hao, R. Zhang, "Analysis of the simple control system of a Multiple-launching Rocket ", J. Projectiles Rockets Missiles Guid, vol. 26 no. 2, 2006.

[3] X. X. Yao, B. Mo, H. B. Li, "Design of the Testing System for Jet Elements", Proceedings Sino-Russion International Academic Conference, vol. 20-21, pp. 80-85, 2000.
[4] C. X. Yang, B. Mo, H. B. Li, In: Proceedings of the Fourth International Symposium on Test and Measurement , 2001.

[5] ANSYS Inc., GAMBIT Modeling Guide. ANSYS Inc., 2006.

[6] F. Dexun, M. Yanwen, Computational Fluid Dynamics, 1st ed., Beijing: Higher Education Press, 2002.

[7] J. O. Hinze, Turbulence. New York: McGraw Hill, 1975.

[8] T. H. Shih, W. W. Liou, A. Shabbir, Z. Yang, and J. Zhu, "A neweddy-viscosity model for high reynolds number turbulent flows model development and validation", Comput. Fluids, vol. 24, no. 3, pp. 227-238, 1995.

[9] W. C. Reynolds, "Fundamentals of turbulence for turbulence modeling and simulation", Lecture Notes for Von Karman Institute Agard Report No. 755, 1987.

[10] M. Wolfstein, "The velocity and temperature distribution of onedimensional flow with turbulence augmentation and pressure gradient", Int. J. Heat Mass Transfer, vol. 12, pp. 301-318, 1969.

[11] H. C. Chen and V.C. Patel, "Near-Wall Turbulence Models for Complex Flows Including Separation", AIAA J, vol. 26, no. 6, pp. 641-648, 1998

[12] T. Jongen, "Simulation and Modeling of Turbulent Incompressible Flows", PhD thesis, EPF Lausanne, Lausanne, Switzerland, 1992.

Received: October 10, 2010

Revised: December 27, 2010

Accepted: March 03, 2011

(C) Xu et al.; Licensee Bentham Open.

This is an open access article licensed under the terms of the Creative Commons Attribution Non-Commercial License (http://creativecommons.org/licenses/by-nc/3.0/) which permits unrestricted, non-commercial use, distribution and reproduction in any medium, provided the work is properly cited. 